Old Dominion University

ODU Digital Commons

2015

Exploring a Toll Auction Mechanism Enabled by Vehicle-toInfrastructure Technology

Andrew J. Collins

Erika Frydenlund

R. Michael Robinson

Mecit Cetin

Follow this and additional works at: https://digitalcommons.odu.edu/vmasc_pubs

Part of the Economics Commons, Infrastructure Commons, Public Policy Commons, Transportation Commons, and the Transportation Engineering Commons 


\title{
Exploring a Toll Auction Mechanism Enabled by Vehicle-to-Infrastructure Technology
}

\author{
Andrew J. Collins, Erika Frydenlund, R. Michael Robinson, and Mecit Cetin
}

High-occupancy toll (HOT) lanes—an increasingly popular solution for congested roadway networks-give drivers the option to access express lanes. The cost of entry often varies with demand, although no standard method of optimizing these price points exists. Using the principles of a Vickrey auction that incentivizes true-value bids, this paper proposes a tolling system that uses vehicle-to-infrastructure technology to optimize toll operator revenue with HOT lane usage. In the scenario, a roadway network consists of a HOT lane and a general-purpose lane, each with identical physical properties. Drivers can access the HOT lane at the start of the facility or at one interim point along the roadway. With the use of a triangular distribution to approximate the distribution of travelers' value of time (VOT), the model explores the impact of varying the distribution's mode on revenue earned by the toll operator. Results from the model indicate that when the toll operator maximizes the model's revenue, the percentage of auction bids accepted for toll road access is robust to changes in the VOT distribution. This percentage equates to approximately $17 \%$ of vehicles accessing the facility. Given the difficulty in obtaining actual travelers' distribution of VOT, this auction tolling mechanism implies that obtaining an exact VOT distribution may not be necessary for this type of tolling analysis.

Wireless technology increasingly incorporates everyday appliances such as televisions, refrigerators, and even doorbells. Personal vehicles are no exception to this wireless expansion, or "Internet of things," with companies like OnStar offering emergency and security vehicleto-infrastructure (V2I) capabilities over cellular phone networks. The evolving nature of V2I technology expands the possibilities for new applications, such as the participation of travelers in auctions to access toll roads in which bids are placed via V2I devices (1). This paper considers such an application in a simple traffic scenario with dynamic auction tolling. The mathematical model developed here shows the impact of varying the distribution of travelers' (customers') value of time (VOT) on overall travel time and bid acceptance policies. The results indicate that the proposed auction mechanism is invariant to the VOT distribution and therefore potentially allows future researchers to reduce their concerns about obtaining an exact VOT distribution for tolling models.

The next section gives a brief background on tolling and auctions. An overview of transportation and revenue management theory, which forms the backbone of this analysis, follows. These theories

A.J. Collins, E. Frydenlund, and R.M. Robinson, Virginia Modeling Analysis and Simulation Center, Old Dominion University, 1030 University Boulevard, Suffolk, VA 23435. M. Cetin, Department of Civil and Environmental Engineering, 135 Kaufman Hall, Old Dominion University, Norfolk, VA 23529. Corresponding author: A.J. Collins, ajcollin@odu.edu.

Transportation Research Record: Journal of the Transportation Research Board, No. 2530, Transportation Research Board, Washington, D.C., 2015, pp. 106-113. DOI: $10.3141 / 2530-13$ are then framed as an optimization problem to analyze the distribution of travelers' VOT. The paper concludes by quantifying abstract variables and solving the optimization problem, giving insight into the use of Vickrey auction schemes for dynamic V2I tolling scenarios.

\section{BACKGROUND}

As volumes on transportation networks increase, factors such as environmental constraints, right-of-way issues, societal impacts, and reduced public funding limit the ability and attractiveness of reducing congestion by constructing new roads (2). When new facility expansion and construction are not options, planners must employ alternative strategies. One popular strategy is the use of high-occupancy vehicle (HOV) lanes. Adopting this strategy requires at least two key components, namely, the availability of an extra lane for HOV travel and a way to ensure optimal use of the dedicated lane. To maximize the potential flow improvements provided by HOV lanes, some locales expand the eligible vehicles to include hybrid electric cars and tollpaying customers. HOV lanes combined with tolls are called highoccupancy toll (HOT) lanes. The first HOT lane was implemented in 1995 on SR-91 in Orange County, California (3).

Tolling can help relieve congestion while generating funds for transportation infrastructure improvements and is thus likely to become more prevalent in the future. Poole points out that most major Interstate corridors were built in the 1960s and 1970s with a 50-year life span and are nearing the end of their expected service (4). Poole estimates that revitalizing these roads will cost $\$ 3.14$ trillion over the next 30 years. Tolling might be the only viable means to fund this extensive project, and thus it represents an important facet of transportation research.

With the use of nondisruptive technology (e.g., E-ZPass) that automatically bills the toll road user and eliminates the need for stopping at tollbooths, tolls are becoming easier and more efficient to use. Given the developments in tolling technology and the increasing adoption of this congestion reduction strategy, more research must investigate available tolling policies. Combining the use of mathematical modeling of tolling mechanisms (5-8) with studies on V2I for traffic management (9), this paper examines a scenario in which driverless vehicles allow travelers to bid in a live auction for tolls on HOT lanes by using V2I technology. The following sections provide explanations of current tolling mechanisms and then describe the scenario and research methodology.

\section{Tolling Mechanisms}

Two important elements of modeling tolls are the tolling mechanism and customer utility. The tolling mechanism has traditionally 
taken the form of a fixed cost for using the toll road; customer utility involves a VOT equation with customers' choices modeled in a variety of ways (3).

Traditionally, toll roads have fixed prices. One advantage to this approach is that drivers and customers know what to expect and can prepare for the payment amount. As nondisruptive payment methods, such as E-ZPass, become more common, automated tolling mechanisms reduce the need for tollbooths and introduce the potential for varying toll prices. Dynamic price points for tolls reflect preset time intervals, such as rush hour, or real-time response to congestion. Examples of real-world dynamic tolls include the San Diego I-15 FasTrak, which changes the toll price every 17 min during peak periods; the Orange County, California, SR-91 tolls, which change every hour; the Minnesota I-394 tolls, which change as frequently as every $3 \mathrm{~min}$; and the I-95 express toll lanes in Florida, which change the toll price every $15 \mathrm{~min}(7)$.

An adjustable tolling mechanism allows control over the number of vehicles using the roadway or HOT lane. Setting a toll price too low leads to overutilization, congestion, and degradation in service quality. Setting the price too high will discourage use and lead to underutilization of the HOT lane and less-than-optimal congestion relief for the general-purpose (GP) lanes. This paper contributes to the theoretical and practical study of tolling mechanisms to ensure that an optimal number of vehicles enter the toll lane both to relieve congestion and to maximize revenue (6).

Literature on tolling mechanisms reveals a variety of approaches to maximizing revenue. Cheng and Ishak developed a feedback mechanism for maximizing toll revenue while ensuring that the toll road maintained a minimum speed of $45 \mathrm{mph}$ or higher, which they tested in a PTV VISSIM simulation (7). Zhang et al. initially followed a similar approach (10). Subsequent research by Zhang et al. adapted this approach to include control theory, in which a proportional integral and derivative algorithm was used to control oscillations in flows and to make a smoother ride for toll lane users (8). Wie applied the Stackelberg leadership model from game theory to solve dynamic toll schedules for a specified subset of arcs with bottlenecks on a congested traffic network (5). Shepherd and Balijepalli modeled the use of toll pricing as a means for cities in competition with one another to attract new residents $(11,12)$. Their results indicated that competition resulted in unfavorable tolling strategies for both cities. Friesz et al. presented a sophisticated method for dynamic congestion pricing; however, they faced computationally intensive and difficult implementation (13). The problem of computational intensity, known as the curse of dimensionality, plagues many techniques developed to study congestion pricing. The complex nature of the studies described here highlights the difficulty in optimizing vehicle use of the toll road.

Toll price determination usually focuses on the relationship between customers (travelers) and toll operators. With V2I technology, the potential exists for the toll operator to directly interface with travelers in real time in response to road conditions. The toll operator would respond to higher or lower demand by raising or lowering the price accordingly. Times of high volume, however, leave little room for error in determining the toll price. Setting the price too low could lead to congestion on the toll road beyond that of the GP lanes and, in turn, to customer dissatisfaction. Rather than allowing the toll operator to allocate eligible access to the toll road randomly when demand is higher than capacity, this study proposes the use of an auction to determine which vehicles will pay what price to enter the HOT lane.

The following auction mechanism is proposed to adjust toll amounts dynamically on the basis of travelers' demonstrated valuation of road use provided by V2I technology by placing bids for the maximum amount they are willing to pay to use the toll road, an amount roughly equivalent to their individual value of time. The toll operator then determines a cutoff price for bids on the basis of the number of vehicles that can be admitted to the road without exceeding desired density levels. This version of the auction mechanism is a one-shot auction, but an auction can be run in many different ways. [An overview of standard auction types appears in Teodorović et al. (14) and in Dash et al. (15)]. Different auction mechanisms will result in different revenue payouts to the auctioneer, so tolling mechanisms should adapt to suit the needs of the specific scenario.

This research incorporates the mechanism of a Vickrey auction, which works by allowing the auctioneer to set a cutoff for the number of winning bids and then accepting that number of the highest bids. The final price given to the winners is equal to the highest bid that was not accepted (or slightly above it) (16). The proxy bidding system of the eBay website is similar to a Vickrey auction. The advantage of this auction mechanism is that it disincentivizes outbidding behavior when auction participants attempt to cheat the system. Instead, consumers tend to bid their perceived values of the product, leading to realistic valuation of the auction item, in this case, toll prices. This mechanism design allows for multiple bids from travelers but creates some challenges discussed later.

Although the Nobel Prize-winning economist Williams Vickrey published research on both congestion pricing problems (17) and auction theory (16) in the 1960s, he did not connect the two together at the time. This lack of connection likely resulted from the infeasibility of bidding while driving and the lack of means to organize bid outcomes in real time, problems that modern technology solves. With the potential use of V2I technology and driverless vehicles, conducting a tolling auction during transit is now feasible. Until now, research connecting Vickrey's two groundbreaking ideas has focused on bidding before travel rather than bidding while traveling. For example, Teodorović et al. proposed an auction-based congestion pricing scheme for people to bid on entry to a downtown area in a weeklong period to which the bidders wish to make one or more visits (14). The researchers produced a mixed-integer program problem and solved it by using heuristics. The study did not consider V2I technology.

Recently, Zhou and Saigal considered V2I-facilitated auctions and used a combinatorial auction approach to process bids from an interconnected toll road network (1). Combinatorial auctions allow bidders to bid on different or even multiple but overlapping items, such as an interconnected toll network (18). The mechanism that Zhou and Saigal (1) used is called the VCG named after Vickrey (16), Clarke (19), and Groves (20). The VCG works by first deciding which bids maximize revenue and then determining the bid price. This price is based on the difference between the revenue gained if the bidder's bid was accepted and the theoretical revenue gained if the bid was not accepted (i.e., if a bid was not accepted, then other bids might be accepted instead). The VCG approach is very computationally intensive and is NP-hard (14).

This research connects Vickrey's auction mechanism with V2I technology to optimize revenue and lane usage without requiring excessive computation. This process first requires quantification of the driving behavior factors considered to evaluate efficiency and customer satisfaction. The following section establishes the customer utility aspect of this type of tolling mechanism.

\section{Customer Evaluation of Time}

The majority of tolling research, including this study, uses the concept of VOT for determining how much travelers are willing to pay. VOT is a linear multiplying constant that relates time saved to 
a monetary value. The authors accept that using VOT has several drawbacks, including that it does not take into account the complex factors in human decision making. For example, travelers' VOT reflects time of day, as indicated by the VOT for the I-395 HOT lane in Minnesota that varied from $\$ 73 / \mathrm{h}$ in the morning to $\$ 116 / \mathrm{h}$ in the afternoon (21). Drivers also exhibit a perception bias toward tolls of about 15 to $20 \mathrm{~min}$ for which they will accept an increase in travel time to avoid a toll (22). In addition, research suggests that travelers value time savings more on longer trips (22) and that they are willing to accept time-varying toll charges only if those charges can yield more-reliable journey times (23). These factors could explain why Cho et al. discovered a limited correlation between time saved and proportion of travelers using HOT lanes (21). These human factors indicate that VOT may require the addition of a constant value. Michalaka et al. presented more complex examinations of VOT by using artificial intelligence techniques to learn travelers' dynamic VOT with data from the I-95 Express in Florida, but this issue is beyond the scope of the current study (2).

In this model, VOT is secondary to the auction pricing mechanism, the primary area of inquiry here. For that reason, the inability of VOT to capture more complex human decision-making processes is outweighed by the simplicity of the approach. In this study, the heterogeneity of VOT, rather than the actual function, is the most important aspect of this measure.

\section{Customer Choice Models}

A final aspect of consumer behavior incorporated into the model involves determining how drivers make the choice to participate in dynamic tolling auctions. In the literature on toll participation, the favored approach is to use logit choice models (24) to replicate human decision making about whether to take the toll road $(7,10)$. Gardner et al. did a comparative study of customer choice models and concluded that the Burr distribution was the best fit for customer choice (3). The model in the current paper assumes that every traveler has a value for the time-saving aspect of using the toll road (even if that value is zero), so every vehicle places a bid. This feature means that a traveler's only choice in this model is the amount of his or her bid, which the authors assume is based on that traveler's VOT.

\section{MODEL DESIGN}

The research presented in this paper considers a simple tolling scenario (Figure 1) to investigate the impact of assuming different estimates of VOT distribution on both the toll operator's revenue and the travel times of the vehicles in the system.

\section{Scenario}

The scenario involves a single origin-destination pair, AC, connected by a GP road and a toll road. At the midway point between

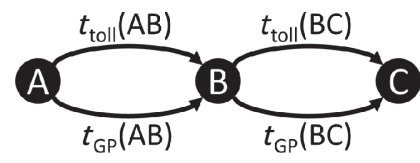

FIGURE 1 Node-arc diagram of scenario transportation network. nodes $\mathrm{A}$ and $\mathrm{C}$ is an interchange $\mathrm{B}$ between the toll road and the GP road (Figure 1). The authors assume that movement between the two roads at the intersection is smooth and does not interfere with the flow of traffic. All segments are identical in length and capacity. Thus, given the same vehicle volume, travel time is the same on all road segments. The total volume of vehicles is normalized to equal one without loss of generality.

Unlike in traditional fixed-tolling mechanisms, travelers in this scenario bid to use the toll road, and the toll operator determines which bids are accepted. The travelers initially place two bids: one for use of the toll road from $\mathrm{A}$ to $\mathrm{C}$ without interruption, $b(\mathrm{AC})$, and one for the toll road from A to $\mathrm{B}, b(\mathrm{AB})$. If a traveler's $b(\mathrm{AC})$ bid is accepted, that traveler's other bids are nullified. Travelers whose initial $b(\mathrm{AC})$ bids are not accepted are provided the opportunity to bid for the BC segment; thus, three potential bidding opportunities are available. A traveler may get to use the toll road for the complete journey even if his $b(\mathrm{AC})$ bid is rejected, as his $b(\mathrm{AB})$ and $b(\mathrm{BC})$ bids might be accepted.

Toll or HOT lane operation policies may have multiple objectives. The most common are to maximize throughput of the entire freeway (both GP and toll lanes) and to provide free-flow traffic service on the toll lane. The authors assume in this scenario that the toll operator's only objective is to maximize revenue from the tolls collected. Thus, the operator is not concerned with minimizing overall travel time or maximizing road efficiency.

This scenario assumes that all vehicles and toll operators have the V2I technology required for this process to occur. In addition, it assumes that all vehicles can place bids before reaching the selection nodes $\mathrm{A}$ and $\mathrm{B}$ and that no slowdown from a vehicle entering or exiting a toll lane occurs. The final assumption is that all travelers are able to honor their bids.

The following sections describe the three parts required to produce a complete toll model: the link travel time function, the bidding mechanism, and the customer's VOT.

\section{Link Travel Time Function}

As VOT is directly related to bids placed by travelers, the time required to transverse the network and the effects of congestion are critical. The U.S. Bureau of Public Roads uses a standard equation for congestion on road segments, shown in Equation 1, the use of which is also supported by Teodorović et al. (14). This equation is based on Greenshields's fundamental diagram of traffic flow (25):

$t(l, v)=t_{\mathrm{ff}}(l)\left(1+0.15\left(\frac{v}{c(l)}\right)^{4}\right)$

where

$$
\begin{aligned}
t & =\text { travel time of link } l \text { for traffic volume } v, \\
t_{\mathrm{ff}} & =\text { free-flow travel time, and } \\
c & =\text { road segment capacity. }
\end{aligned}
$$

Justified by its acceptance in congestion and tolling studies, this equation will also inform model development for this study.

\section{Bidding Mechanism}

Because the entire bidding process in this scenario occurs over wireless V2I technology, this system has two decision makers, the 
traveler and the toll operator. Equations 2 and 3 represent the decisions of travelers. The decision of the toll operator is discussed in the section on the model. On the basis of individual VOT, travelers will place bids for access to toll road segments. The authors assume that travelers have perfect knowledge about travel time on the road segments and are thus able to determine the travel time savings of using the toll road. The justification for this perfect knowledge is the assumption that regularly commuting travelers along the road would likely be able to make accurate estimates of travel time on the basis of current conditions. By using a Vickrey auction mechanism, travelers lack incentive to bid anything other than their true estimates of the toll price. From these assumptions, the bidding formula for travelers for arc $\mathrm{AB}$ in Equation 2 is based on the difference in travel times between the GP and the toll lanes:

$b(\mathrm{AB}, x)=u(x)\left(t\left(\mathrm{AB}_{\mathrm{GP}}, v\left(\mathrm{AB}_{\mathrm{GP}}\right)\right)-t\left(\mathrm{AB}_{\text {toll }}, v\left(\mathrm{AB}_{\text {toll }}\right)\right)\right)$

where $x$ is the traveler and $u$ is the VOT. The advantage of using this bidding equation is that it stops the scenario when the toll operator simply accepts all bids. Such a condition would make the toll road's congestion worse than that of the GP lane and lead travelers to bid zero.

$b(\mathrm{AC}, x)=b(\mathrm{AB}, x)+b(\mathrm{BC}, x)$

A variation of Equation 2 can also be used for bids of the $\mathrm{BC}$ road segment. Determining bids for using the toll road all the way from A to $\mathrm{C}, b(\mathrm{AC})$, is trickier because it involves multiple road segments (Equation 3). The authors assume that this bid considers only travel time savings and therefore means that it relates to Equation 2. This estimate of the AC bid may be problematic and will be discussed in the section on results.

\section{Travelers' VOT Distribution}

The model assumes that travelers propose heterogeneous bids; otherwise, everyone would bid the same amount. Here a method for modeling travelers' VOT is established. This step means that each traveler will have a different VOT defined as $u(x)$ in Equation 2. Thus, the distribution of the traveler's VOT needs to be defined. Without any estimate of the distribution of VOT, the model leans toward parsimony and applies a simple triangular distribution. The triangular distribution allows the setting of a minimum VOT (i.e., zero) and a maximum VOT (as even billionaires have limits on their VOT). Unlike the uniform distribution, the triangular distribution also allows the setting of an average, the mode. Then this mode can be varied to produce different distributions (Figure 2). Other functions, like the beta function, achieve this variability but require more complex implementation. The triangular function is used in other transportation models as an approximation of relationships like flow to density (2). Because the function is continuous, the authors assume that the number of vehicles is continuous and not discrete. To simplify the mathematics, the number of vehicles is normalized to one.

\section{MODEL}

Here the model is developed that helps the second set of decision makers, the toll operators, who decide which bids to accept given the travelers' bidding strategies discussed earlier. Because of the structure of Vickrey auctions, the accepted price for toll road access

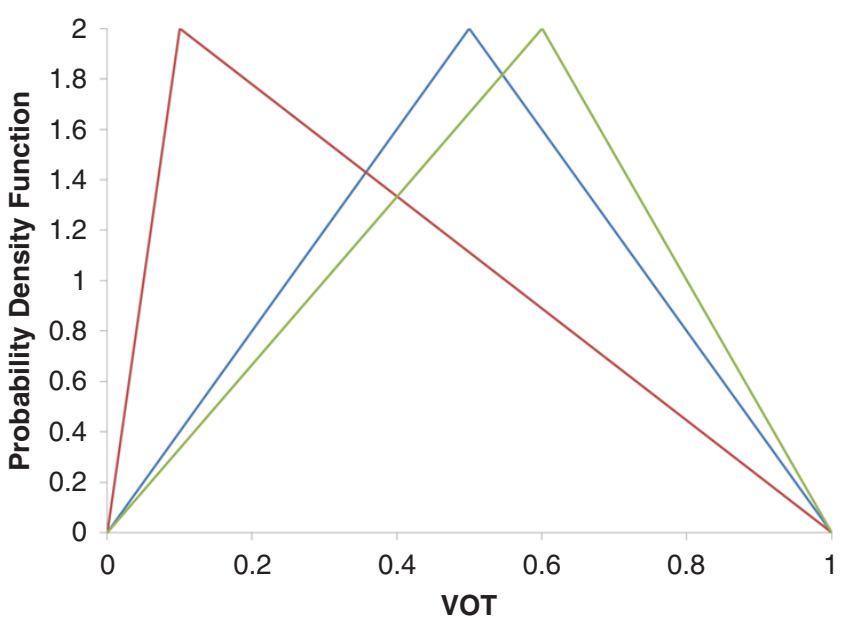

FIGURE 2 Various versions of triangular distribution with minimum of zero and maximum of one.

is the value of the lowest accepted bid-or highest nonaccepted bid in the continuous case. The toll operator then faces a dilemma: as more vehicles are accepted, the number of payments collected increases, but the toll price for each is lower. The toll operator must balance the number of vehicles accepted against the actual toll price paid. In addition, the more vehicles that are accepted onto the toll road, the less attractive it is to drivers (more vehicles mean slower travel times), so they will lower their initial bids. The toll operator must consider these factors to maximize toll revenue.

The toll operator must make three decisions:

- The number of bids to accept for use of the entire length of the toll road, $b(\mathrm{AC})$;

- The number of bids to accept for only the first toll segment, $b(\mathrm{AB})$; and

- The number of bids to accept for only the last toll segment, $b(\mathrm{BC})$.

The authors assume that the toll operator takes the highest bids available for each segment and that the operator prefers bids for $b(\mathrm{AC})$ over those for $b(\mathrm{AB})$, as more guaranteed revenue is generated. From these assumptions the following optimization problem is developed:

$$
\begin{aligned}
\max _{\theta, \mu, \lambda \in[0,1]} & \int_{F^{-1}(1-\lambda)}^{u_{\max }} b\left(\mathrm{AC}, F^{-1}(1-\lambda)\right) f(x) d x \\
+ & \int_{F^{-1}(1-(\theta+\lambda))}^{F^{-1}(1-\lambda)} b\left(\mathrm{AB}, F^{-1}(1-(\theta+\lambda))\right) f(x) d x \\
+ & \int_{F^{-1}(1-(\mu+\lambda))}^{F^{-1}(1-\lambda)} b\left(\mathrm{BC}, F^{-1}(1-(\mu+\lambda))\right) f(x) d x
\end{aligned}
$$

such that

$\theta+\lambda \leq 1$

and

$\mu+\lambda \leq 1$ 
where

$\lambda=$ proportion of travelers whose $b(\mathrm{AC})$ bid is accepted,

$\theta=$ proportion of travelers whose $b(\mathrm{AB})$ bid is accepted,

$\mu=$ proportion of travelers whose $b(\mathrm{BC})$ bid is accepted,

$F=$ cumulative distribution function $(\mathrm{CDF})$ of the triangular distribution,

$f=$ probability distribution function (PDF) of the triangular distribution,

$u_{\max }=$ upper bound of the triangular distribution, and

$u_{\text {mod }}=$ distribution's mode.

The vehicles are identified by their associated VOT; thus, $u(x)=x$ here; because the vehicles are represented as continuous variables, their VOTs are unique. The constraints ensure that no more vehicles are allocated to the arc $\mathrm{AB}_{\text {toll }}$ and $\mathrm{BC}_{\text {toll }}$ than the number of vehicles in the system (which was normalized to one). The three integrals show the total revenue generated from the three groups: those accepted for travel on the complete toll road and those accepted for travel on only one of the two segments. The price paid by each vehicle in a particular group is constant and is equal to the lowest bid offered for that group. Thus, the equation can be simplified to the following:

$$
\begin{aligned}
& \max _{\theta, \mu, \lambda \in[0,1]} \lambda b\left(\mathrm{AC}, F^{-1}(1-\lambda)\right)+\theta b(\left.\mathrm{AB}, F^{-1}(1-(\theta+\lambda))\right) \\
&+\mu b\left(\mathrm{BC}, F^{-1}(1-(\mu+\lambda))\right)
\end{aligned}
$$

Before the optimization equation is further manipulated, some simplifying assumptions are made. The authors have assumed that all the arcs are identical, and the free-flow travel is normalized to get $\mathrm{T}_{f f}\{\cdot\}=1$. The travel time equation requires the volume of traffic, which is assumed to be just the proportion of vehicles that use that particular arc. This assumption means that discrete traffic flow is also assumed as is the fact that the number of vehicles is continuous. Further assumed is that capacity is $c\{\cdot\}=1$, which means that no arc can have more than the full capacity of vehicles flowing through it. On the basis of these assumptions, Equation 5 becomes the following:

$$
\begin{aligned}
\max _{\theta, \mu, \lambda \in[0,1]} 0.15( & \left.\lambda F^{-1}(1-\lambda)+\theta F^{-1}(1-(\theta+\lambda))\right) \\
& \left(\left((1-(\theta+\lambda))^{4}-(\theta+\lambda)^{4}\right)\right)+0.15\left(\lambda F^{-1}(1-\lambda)\right. \\
& \left.+\mu F^{-1}(1-(\mu+\lambda))\right)\left(\left((1-(\mu+\lambda))^{4}-(\mu+\lambda)^{4}\right)\right)
\end{aligned}
$$

Equation 6 reduces the number of additive parts of Equation 5 from three to two; this reduction occurs because of the relation of the bids given in Equation 3. At this stage, Equation 6 could use any probability distribution for the travelers' VOT. The final stage of the manipulation of the optimization equation is to substitute the triangular distribution into it. The triangular distribution's cumulative distribution function is continuous but involves different functional parts for each side of the mode value. To keep the equations simple, the following are assumed:

$$
\begin{aligned}
& F^{-1}(1-(\theta+\lambda)) \geq u_{\mathrm{mod}} \\
& F^{-1}(1-(\mu+\lambda)) \geq u_{\mathrm{mod}}
\end{aligned}
$$

Under these constraints, one side of the triangular distribution needs to be considered. Thus

$$
F^{-1}(1-x)=u_{\max }-\sqrt{x \cdot u_{\max }\left(u_{\max }-u_{\bmod }\right)}
$$

This arrangement means that the new constraints become

$$
\begin{aligned}
& (\theta+\lambda) \leq \frac{\mu_{\max }-u_{\mathrm{mod}}}{u_{\max }} \\
& (\mu+\lambda) \leq \frac{\mu_{\max }-u_{\bmod }}{u_{\max }}
\end{aligned}
$$

Substituting Equation 8 into Equation 6 results in the following optimization problem, with the constraints above:

$$
\begin{array}{r}
\max _{\theta, \mu, \lambda \in[0,1]} 0.15\left(\begin{array}{l}
(\theta+\lambda) u_{\max }-\sqrt{u_{\max \left(u_{\max }-u_{\bmod }\right)}} \\
(\lambda \sqrt{1-\lambda}+\theta \sqrt{1-(\theta+\lambda)})
\end{array}\right)\left(\left(\begin{array}{c}
(1-(\theta+\lambda))^{4} \\
-(\theta+\lambda)^{4}
\end{array}\right)\right) \\
+0.15\left(\begin{array}{l}
(\mu+\lambda) u_{\max }-\sqrt{u_{\max \left(u_{\max }-u_{\bmod }\right)}} \\
(\lambda \sqrt{1-\lambda}+\mu \sqrt{1-(\mu+\lambda)})
\end{array}\right)\left(\left(\begin{array}{c}
(1-(\mu+\lambda))^{4} \\
-(\mu+\lambda)^{4}
\end{array}\right)\right)
\end{array}
$$

\section{RESULTS}

The equation was numerically solved by using the NMaximize function in Mathematica 10 (www.wolfram.com/mathematica/), which was set at a precision of five significant figures of accuracy. The NMaximize function runs various heuristic methods to find the solution to the level of accuracy required. These methods include simulated annealing (26) and the Nelder-Mead method (27). Using this heuristic, the authors explored the effects of varying the VOT triangular distribution on model results. This exploration was achieved by varying the $u_{\text {mod }}$ value from 0.0 to 0.9 . The results are shown in Figure 3. Several aspects of the results are noteworthy and will be discussed in turn.

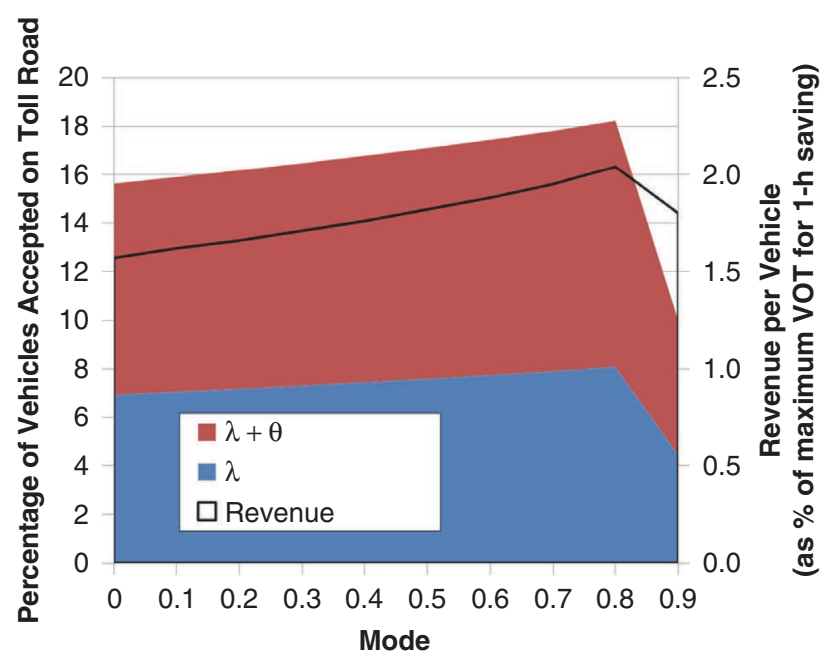

FIGURE 3 Graph showing results from varying mode value of VOT distribution for both revenue generated and proportion of vehicles allowed on toll road. 
Varying the $u_{\text {mod }}$ value produces very different distributions. For example, the triangular distribution with a $u_{\bmod }=0.9$ is the opposite (mirror image) of one with a $u_{\text {mod }}=0.1$. Given these differences, the numerical solutions showed surprisingly similar results, except for $u_{\text {mod }}>0.8$. The argument solution to all these cases was approximately $\lambda=0.076, \theta=0.095, \mu=0.095$ with a revenue generated of 0.02 . The argument solutions translate to the percentage of vehicles allowed on the toll road, which is approximately $17 \%$ on both toll segments. The revenue is a little more complex to understand and requires an explicit example: consider a situation in which each toll segment takes a half hour to travel at free-flow speed and maximum VOT is $\$ 100 / \mathrm{h}$ saved. Then the revenue generated per 6,000 vehicles entering the system (either on the toll road or the GP lanes) is $\$ 12,000$ (by assuming that capacity is greater than 6,000 vehicles), with an average charge of approximately $\$ 11$ per vehicle.

The trend does not continue for $u_{\text {mod }}>0.8$ because of an analytical limitation placed on the model. The authors assumed (to simplify the equations) that only vehicles whose VOT $>u_{\text {mod }}$ would be considered for acceptance, an assumption that resulted in some constraints given in Equation 9. When $u_{\text {mod }}<0.8$, these constraints hold for the optimal solution; however, as $u_{\text {mod }}$ increases beyond this point, these constraints limit the number of vehicles whose bids can be accepted and thus result in a reduction in overall revenue and number of vehicles accepted.

It might have been expected that the toll operator would only accept $\mathrm{AC}$ bids and not any bids from $\mathrm{AB}$ or $\mathrm{BC}$. However, accepting bids from both groups allows the toll operator to get a higher toll price for the AC group (in accordance with the Vickrey auction mechanism) in addition to the revenue generated by the $\mathrm{AB}$ and $\mathrm{BC}$ groups. Thus, the toll operator can charge more to those willing to pay more for complete access to both toll road segments as well as make money from secondary customers. This strategy is quite common in airline pricing, for which a large difference usually exists between the price of a business class ticket and an economy class ticket (24).

For all $u_{\text {mod }}, \theta=\mu$, which implies that the same group of vehicles gets accepted on the toll road from $\mathrm{AB}$ as those on the toll road from $\mathrm{BC}$, thus effectively getting the same benefit as those whose $\mathrm{AC}$ bids were accepted. This result is unsurprising, as the selection situation is the same for both $\mathrm{AB}$ and $\mathrm{BC}$ : the toll and GP lanes are exactly the same; the number of vehicles on the toll is the same (from those who have been selected from the AC bids); and the bids are exactly the same (as it is the same group of travelers remaining in both cases). However, the first group pays $80 \%$ of the maximum bid, and the second group pays $70 \%$ of the maximum; thus, the group guaranteed complete access to the two toll road segments pays a $10 \%$ premium. This result could lead to complications from a game theory point of view because the top percentage of travelers would benefit from not bidding on the AC option. However, all travelers accepted on the toll road pay less than their VOT saved, so they would still benefit from the current arrangement.

Given that $\theta=\mu$ means that $\lambda+\theta=\lambda+\mu$, only one of these values is shown in Figure 3. The graph shows a slight increase in the percentage of vehicles accepted to use the toll road as $u_{\text {mod }}$ increases. As $u_{\text {mod }}$ increases the traveler's willingness to accept a higher price increases, so one might expect more vehicles to be accepted on the toll road, and they are. However, acceptance of more vehicles slows the travel time and reduces the benefit of the toll road. This change motivates those travelers with a higher VOT to sub- mit lower bids. This trade-off forces the toll operator to perform, because of the changing bids, a balancing act between the number of cars accepted on the toll road and the amount of potential revenue per car.

With approximately $17 \%$ of vehicles using the toll road, travel time reaches just over free-flow speed (i.e., 1.00004), whereas the GP lane users see a $70 \%$ increase in travel time because of congestion. For the hour journey of the hypothetical scenario, these differences mean that the toll users save 42 min for about $\$ 11$.

A noticeable feature of Figure 3 is the invariance of the results - in both accepted toll users and in revenue obtained by the toll operatorto extreme changes in the VOT distribution. An approximate $10 \%$ increase remains in both accepted toll users and revenue obtained between the extremes, results that are expected (if users have a lower average VOT lower revenues would be expected). At this stage of the research, the authors are not sure whether the phenomenon represents reality or is a consequence of the model assumptions (e.g., the use of a triangular distribution, which is inherently linear, like the observed increase in revenues). Further research is needed to determine the robustness of this observation to varying modeling assumptions and comparison with real-world data.

\section{DISCUSSION OF APPLICATION}

The discussion so far has been academic in nature and, as such, has not considered the practical limitations of implementing such a toll auction mechanism. Four practical limitations come to mind: education, participation rates, perception, and the dead-fish fallacy.

Existing variable tolling mechanisms may be more intuitive than the one proposed here. Though the proposed bidding mechanism is simpler than other auction types, it is still a combinatorial auction and its mechanics might be beyond the grasp of the general population. From a practical perspective, the traveler must understand that multiple bids happen simultaneously and correspond only to a certain segment or segments of the roadway network. In addition, placing a bid does not guarantee acceptance to the toll road, as only the selected top bidders gain access. In addition to the ubiquitous spread of V2I technology that would be required to implement this proposed tolling mechanism, the toll operator would likely need to pursue an extensive advertising and public relations campaign before deployment.

The current model assumes $100 \%$ participation rates, which are highly unlikely among the general population, especially for those with a low VOT. In practice, the V2I technology and tolling mechanism would need a component to allow for default bid values. These values would allow travelers to set a very minimal bid that they are always willing to pay for use of the toll road. The current optimization model does not account for this option. The impact of default bids on an auction-based tolling mechanism is an area for further study.

An auction-based system for HOT lanes could result in negative public perception. Ethical considerations for who has access to the toll or which bids are accepted constitute a hurdle for HOT implementation in general. Given that accepted bids reflect the highest losing bid, uninformed travelers may be skeptical of the method for selecting winning bids as prices constantly vary.

The authors' final concern is that this system assumes a steady state because the drivers in the scenario have both perfect knowledge 
and a constant VOT that is not affected by the system. The authors specifically made these assumptions to explore the mathematical theorizing of a Vickrey auction tolling system, but they do not reflect real-world human dynamics that are constantly in flux. Salt calls this the dead-fish fallacy, which highlights the idea that, despite the nonstatic, dynamic nature of the world, scientists insist on assuming static behavior (28). This assumption has consequences on the results drawn: experimental, observational, or both. To illustrate this, Salt colorfully draws a parallel by noting that the only time that fish remain static in a pond is when they are all dead. In the case of a Vickrey auction tolling mechanism, the authors submit to the dead-fish fallacy to assume that a steady state is an approximation for the long-term behavior of the tolling system but not for the initial implementation term. Finally, the authors assume that travelers' VOTs are not affected by the system, an assumption that may be unreasonable in the real world. For example, if a traveler is willing to pay $\$ 10$ to use a toll road, but the final price consistently falls to $\$ 1$, then might that traveler question why his or her VOT is so high? The market itself, and people's own social networks, influence their VOT and may bring the overall revenue potential down (29). As technology advances to make the proposed tolling mechanism more feasible, more research is required to address these limitations and account for human heterogeneity of VOT and decision criteria to ensure optimal revenue and lane usage.

\section{CONCLUSIONS}

This paper describes and models a tolling scenario in which automotive travelers use V2I technology to participate in a Vickrey auction for access to the toll-HOT lane. Access to the toll road occurs at multiple points along the single facility, and travelers are able to make multiple bids to gain access to all or parts of the toll lane. The paper uses this model to explore the impact of varying the distribution of travelers' VOT on the revenue achieved by the toll operator. This VOT was approximated with a triangular distribution for which mode value was the independent variable. The results indicate robustness of the auction mechanism design to variation in the distribution of travelers' VOT. This variation affected the revenue obtained by the toll operator and the number of vehicles accepted on the toll road, which was about $17 \%$ of the total vehicles entering the system.

The advantage of using V2I tolling is that the toll operator can dynamically adjust the toll price to ensure full utilization of the toll road. In the model here, however, road utilization maximizes the toll operators' revenue without necessarily attempting to provide the best option for travelers. The socially optimal solution, in average travel time, would be to allow equal numbers of cars on the toll lane as the GP lane. Doing so would remove the benefit of using the toll lane over the GP lane and would thus disincentivize bidding and reduce the toll operator's revenue to zero. Therefore, an incentive exists for the toll operator to keep the GP lane congested, a practice that follows the old business adage of creating demand for one's product. Because travelers bid on use of the toll road, their doing so means that their satisfaction with the toll operator's acceptance strategy is implicitly included in their bid. This system keeps the toll operator interested only in the paying travelers. Those who win the Vickrey auction are the ones with the highest VOT (i.e., the richest). This system therefore leads to some embedded socioeconomic inequalities that surface through the use of this tolling strategy.

If this proposed system is any reflection of reality, then its invariance to the population's distribution of VOT indicates that auction-based tolling could be an unnecessary mechanism because the population makeup and preferences have very little effect on the outcome. Traditional dynamic tolling mechanisms possibly perform at least as well as the Vickrey auction version, but further research is required to determine whether the model results presented here truly reflect reality.

\section{ACKNOWLEDGMENT}

This project was funded by the National Transportation Center at Maryland (NTC@Maryland), one of five national centers selected in a nationwide competition by the Office of the Assistant Secretary for Research and Technology, U.S. Department of Transportation.

\section{REFERENCES}

1. Zhou, H., and R. Saigal. Combinatorial Auction to Allocate Traffic. In Transportation Research Record: Journal of the Transportation Research Board, No. 2423, Transportation Research Board of the National Academies, Washington, D.C., 2014, pp. 35-43.

2. Michalaka, D., Y. Lou, and Y. Yin. Proactive and Robust Dynamic Pricing Strategies for High-Occupancy Toll (HOT) Lanes. Presented at 90th Annual Meeting of the Transportation Research Board, Washington, D.C., 2011.

3. Gardner, L., H. Bar-Gera, and S. Boyles. Evaluation Framework for High-Occupancy Toll Lanes. Presented at 92nd Annual Meeting of the Transportation Research Board, Washington, D.C., 2013.

4. Poole, R. W., Jr. Modernizing the U.S. Interstate Highway System via Toll Finance. Presented at 93rd Annual Meeting of the Transportation Research Board, Washington, D.C., 2014.

5. Wie, B.-W. Dynamic Stackelberg Equilibrium Congestion Pricing. Transportation Research Part C: Emerging Technologies, Vol. 15, No. 3, 2007 , pp. 154-174.

6. Yang, F. Day-to-Day Dynamic Optimal Tolls with Elastic Demand. Presented at 87th Annual Meeting of the Transportation Research Board, Washington, D.C., 2008.

7. Cheng, D., and S. Ishak. Dynamic Feedback-Control Toll Pricing Methodology for Revenue Maximization: Case Study on I-95 Managed Lanes. Presented at 92nd Annual Meeting of the Transportation Research Board, Washington, D.C., 2013.

8. Zhang, G., X. Ma, and Y. Wang. Self-Adaptive Tolling Strategy for Enhanced High-Occupancy Toll Lane Operations. Transactions on Intelligent Transportation Systems, Vol. 15, No. 1, 2014, pp. 306-317.

9. Milanés, V., J. Villagra, J. Godoy, J. Simó, J. Pérez, and E. Onieva. An Intelligent V2I-Based Traffic Management System. Transactions on Intelligent Transportation Systems, Vol. 13, No. 1, 2012, pp. 49-58.

10. Zhang, G., Y. Wang, H. Wei, and P. Yi. A Feedback-Based Dynamic Tolling Algorithm for High-Occupancy Toll Lane Operations. In Transportation Research Record: Journal of the Transportation Research Board, No. 2065, Transportation Research Board of the National Academies, Washington, D.C., 2008, pp. 54-63.

11. Shepherd, S., and C. Balijepalli. A Dynamic Model of Two Competing Cities: The Effects of Competition on Tolls and Land Use. Proc., 30th International Conference of the System Dynamics Society, St. Gallen, Switzerland, 2012

12. Shepherd, S., and N. C. Balijepalli. Effects of Competition Between Two Neighboring Cities on Tolls and Land Use. Presented at 92nd Annual Meeting of the Transportation Research Board, Washington, D.C., 2013.

13. Friesz, T.L., C. Kwon, and R. Mookherjee. A Computable Theory of Dynamic Congestion Pricing. Proc., 17th International Symposium on Traffic and Transportation Theory, London, 2007, pp. 1-26. 
14. Teodorović, D., K. Triantis, P. Edara, Y. Zhao, and S. Mladenović. AuctionBased Congestion Pricing. Transportation Planning and Technology, Vol. 31, No. 4, 2008, pp. 399-416.

15. Dash, R.K., N.R. Jennings, and D.C. Parkes. ComputationalMechanism Design: A Call to Arms. Intelligent Systems, Vol. 18, No. 6, 2003, pp. 40-47.

16. Vickrey, W. Counterspeculation, Auctions, and Competitive Sealed Tenders. Journal of Finance, Vol. 16, No. 1, 1961, pp. 8-37.

17. Vickrey, W. S. Congestion Theory and Transport Investment. American Economic Review, Vol. 59, No. 2, 1969, pp. 251-260.

18. Cramton, P., Y. Shoham, and R. Steinberg, eds. Combinatorial Auctions, MIT Press, Cambridge, 2006.

19. Clarke, E. H. Multipart Pricing of Public Goods. Public Choice, Vol. 11, No. 1, 1971, pp. 17-33.

20. Groves, T. Incentives in Teams. Econometrica: Journal of the Econometric Society, 1973, pp. 617-631.

21. Cho, Y., R. Goel, P. Gupta, G. Bogonko, and M. W. Burris. What Are I-394 HOT Lane Drivers Paying for? Presented at 90th Annual Meeting of the Transportation Research Board, Washington, D.C., 2011.

22. Koppelman, F. SHRP 2 Report S2-C04-RW-1: Improving Our Understanding of How Highway Congestion and Price Affect Travel Demand.
Transportation Research Board of the National Academies, Washington, D.C., 2013.

23. Bonsall, P., J. Shires, J. Maule, B. Matthews, and J. Beale. Responses to Complex Pricing Signals: Theory, Evidence, and Implications for Road Pricing. Transportation Research Part A: Policy and Practice, Vol. 41, No. 7, 2007, pp. 672-683.

24. Talluri, K. T., and G. J. van Ryzin. Theory and Practice of Revenue Management. Springer-Verlag, New York, 2004.

25. Greenshields, B.D. A Study of Traffic Capacity. Highway Research Board Proceedings, Vol. 14, 1935, pp. 448-477.

26. van Laarhoven, P. J., and E. H. Aarts. Simulated Annealing: Theory and Applications. Springer, Berlin, 1987.

27. Nelder, J.A., and R. Mead. A Simplex Method for Function Minimization. Computer Journal, Vol. 7, No. 4, 1965, pp. 308-313.

28. Salt, J.D. The Seven Habits of Highly Defective Simulation Projects. Journal of Simulation, Vol. 2, No. 3, 2008, pp. 155-161.

29. Seiler, M., A. J. Collins, and N. H. Fefferman. Strategic Mortgage Default in the Context of a Social Network: An Epidemiological Approach. Journal of Real Estate Research, Vol. 35, No. 4, 2013, pp. 445-475.

The Standing Committee on Congestion Pricing peer-reviewed this paper. 